PROCEEDINGS OF THE

AMERICAN MATHEMATICAL SOCIETY

Volume 141, Number 9, September 2013, Pages 3037-3048

S 0002-9939(2013)11774-0

Article electronically published on June 3, 2013

\title{
VARIATION OF HILBERT COEFFICIENTS
}

\author{
LAURA GHEZZI, SHIRO GOTO, JOOYOUN HONG, AND WOLMER V. VASCONCELOS
}

(Communicated by Irena Peeva)

\begin{abstract}
For a Noetherian local ring $(\mathbf{R}, \mathfrak{m})$, the first two Hilbert coefficients, $e_{0}$ and $e_{1}$, of the $I$-adic filtration of an $\mathfrak{m}$-primary ideal $I$ are known to code for properties of $\mathbf{R}$, of the blowup of $\operatorname{Spec}(\mathbf{R})$ along $V(I)$, and even of their normalizations. We give estimations for these coefficients when $I$ is enlarged (in the case of $e_{1}$ in the same integral closure class) for general Noetherian local rings.
\end{abstract}

\section{INTRODUCTION}

Let $(\mathbf{R}, \mathfrak{m})$ be a Noetherian local ring of dimension $d \geq 1$, and let $I$ be an $\mathfrak{m}$-primary ideal. We will consider multiplicative, decreasing filtrations of $\mathbf{R}$ ideals,

$$
\mathcal{A}=\left\{I_{n} \mid I_{0}=\mathbf{R}, I_{n+1}=I I_{n}, \forall n \gg 0\right\},
$$

integral over the $I$-adic filtration, conveniently coded in the corresponding Rees algebra and its associated graded ring,

$$
\mathcal{R}(\mathcal{A})=\bigoplus_{n \geq 0} I_{n}, \quad \operatorname{gr}_{\mathcal{A}}(\mathbf{R})=\bigoplus_{n \geq 0} I_{n} / I_{n+1} .
$$

Let $\overline{\mathcal{R}}=\bigoplus_{n \geq 0} \overline{I^{n}}$ be the integral closure of the Rees algebra $\mathcal{R}=\mathcal{R}(I)$ with $I_{n}=I^{n}$ for all $n \geq 0$, which we assume to be finite over $\mathcal{R}$.

We will consider the Hilbert coefficients $e_{i}(I)$ associated to $\mathfrak{m}$-primary ideals $I$, for $i=0,1$. These integers play important roles in the corresponding blowup algebras. Some of these issues have a long tradition in the context of CohenMacaulay local rings, but others are of a recent vintage for general Noetherian local rings. From the several problem areas, we highlight the following:

(i) the comparison between $e_{0}$ and $e_{1}$;

(ii) $e_{1}$ and normalization;

(iii) the structure of $\mathbf{R}$ associated to the values of $e_{1}$;

(iv) a variation of $e_{i}$, that is, how $e_{i}(I)$ changes when $I$ is enlarged.

We are concerned here with the last item but give brief comments on the others first.

Received by the editors August 22, 2011 and, in revised form, December 3, 2011.

2010 Mathematics Subject Classification. Primary 13A30; Secondary 13B22, 13H10, 13 H15.

The first author was partially supported by a grant from the City University of New York PSC-CUNY Research Award Program-41.

The second author was partially supported by Grant-in-Aid for Scientific Researches (C) in Japan (19540054) and by a grant from MIMS (Meiji Institute for Advanced Study of Mathematical Sciences).

The fourth author was partially supported by the NSF. 
(i) For Cohen-Macaulay rings, a uniform bound for $e_{1}(I)$ first appeared for rings of dimension 1 in the work of D. Kirby $(\underline{K})$,

$$
e_{1}(\mathfrak{m}) \leq\left(\begin{array}{c}
e_{0}(\mathfrak{m}) \\
2
\end{array}\right)
$$

Progressively, quadratic bounds of this type were developed for arbitrary $\mathfrak{m}$-primary ideals in all dimensions by several authors. As a basic source, RV2 has a systematic development of these formulas along with a comprehensive bibliography. It also treats more general filtrations which were helpful to us. Among the formulas which more directly influenced the authors here, we single out two developed in the work of J. Elias ([E1, E2 $]$ ) and M. E. Rossi and G. Valla ([RV1, RV2] $)$. For an $d$-dimensional Cohen-Macaulay local ring and an $\mathfrak{m}$-primary ideal $I$, the first bound asserts that if $I$ is minimally generated by $m=\nu(I)$ elements,

$$
e_{1}(I) \leq\left(\begin{array}{c}
e_{0}(I) \\
2
\end{array}\right)-\left(\begin{array}{c}
m-d \\
2
\end{array}\right)-\lambda(\mathbf{R} / I)+1 .
$$

The other bound uses the $\mathfrak{m}$-adic order of $I$, that if $I \subset \mathfrak{m}^{s}$ and $\bar{I} \neq \overline{\mathfrak{m}^{s}}$, then

$$
e_{1}(I) \leq\left(\begin{array}{c}
e_{0}(I)-s \\
2
\end{array}\right)
$$

Recently, K. Hanumanthu and C. Huneke ( $[\mathrm{HH}]$ ) brought a new parameter to bear on these formulas with their proof that

$$
e_{1}(I) \leq\left(\begin{array}{c}
e_{0}(I)-k \\
2
\end{array}\right)
$$

where $k$ is the maximal length of chains of integrally closed ideals between $I$ and $\mathfrak{m}$.

(ii) Since $e_{1}(I) \leq \bar{e}_{1}(I):=e_{1}(\overline{\mathcal{R}})$, bounds with a different character arise. A baseline is the fact that when $\mathbf{R}$ is analytically unramified but not necessarily Cohen-Macaulay, one has $\bar{e}_{1}(I) \geq 0$ (GHM] $)$. An upper bound for $\bar{e}_{1}(I)$ (see PUV] for other bounds) is the following. Let $(\mathbf{R}, \mathfrak{m})$ be a reduced Cohen-Macaulay local ring of dimension $d$, essentially of finite type over a perfect field, and let $I$ be an $\mathfrak{m}$-primary ideal. Let $\delta$ be a regular element of the Jacobian ideal of $\mathbf{R}$. Then

$$
e_{1}(I) \leq \bar{e}_{1}(I) \leq \frac{t}{t+1}\left[(d-1) e_{0}(I)+e_{0}((I+\delta \mathbf{R}) / \delta \mathbf{R})\right]
$$

where $t$ is the Cohen-Macaulay type of $\mathbf{R}$. In particular, if $\mathbf{R}$ is a regular local ring,

$$
\bar{e}_{1}(I) \leq \frac{(d-1) e_{0}(I)}{2} .
$$

(iii) When $\mathbf{R}$ is not Cohen-Macaulay, the issues become less structured since the values of $e_{1}(I)$ may be negative. In fact, using the values of $e_{1}(I)$ for ideals generated by systems of parameters led to the characterization of several properties (CohenMacaulay, Buchsbaum, finite cohomology) of the ring $\mathbf{R}$ itself (see [GhGHOPV], GhHV], GO, MV], V2]).

(iv) We shall now outline the main results of this note. (We refer to [V1] for basic definitions and Rees algebras theory.) Sections 2 and 3 are organized around a list of questions about the changes that $e_{0}(I)$ and $e_{1}(I)$ undergo when $I$ varies. An important case is

$$
e_{0}(J), e_{1}(J) \longrightarrow e_{0}(I), e_{1}(I), \quad I=(J, x) .
$$


Clearly the optimal baseline is that of an ideal $J$ generated by a system of parameters, but we will consider very general cases. As will be seen, some relationships involve the multiplicity $f_{0}(J)$ of the special fiber. To describe one of these estimates, let $(\mathbf{R}, \mathfrak{m})$ be a Noetherian local ring of dimension $d \geq 1$, let $J$ be an $\mathfrak{m}$-primary ideal and let $I=\left(J, h_{1}, \ldots, h_{m}\right)$ be integral over $J$ of reduction number $s=\operatorname{red}_{J}(I)$. Then Theorem 2.6 asserts that

$$
e_{1}(I)-e_{1}(J) \leq \lambda(\mathbf{R} /(J: I)) \cdot\left[\left(\begin{array}{c}
m+s \\
s
\end{array}\right)-1\right] \cdot f_{0}(J),
$$

where $f_{0}(J)$ is the multiplicity of the special fiber of $\mathcal{R}(J)=\bigoplus_{n>0} J^{n}$. We add a word of warning in reading some of the formulas with terms like $e_{1}(I)-e_{1}(J)$. When $J$ is a minimal reduction of $I, e_{1}(J)$ is always non-positive, according to [MSV], and vanishes when $\mathbf{R}$ is Cohen-Macaulay. In fact, for unmixed local rings the vanishing characterizes Cohen-Macaulayness ([GhGHOPV]).

In Section 3, we address the need to link the value of $\operatorname{red}_{J}(I)$ to other properties of $J$. This is a well-known fact when $\mathbf{R}$ is a Cohen-Macaulay ring, but we give a general formulation in Theorem 3.3 , Let $(\mathbf{R}, \mathfrak{m})$ be a Noetherian local ring of dimension $d \geq 1$ and infinite residue field. For an $\mathfrak{m}$-primary ideal $I$ and a minimal reduction $J$ of $I$, there exists a minimal reduction $Q$ of $I$ such that

$$
\operatorname{red}_{Q}(I) \leq \max \{d \cdot \lambda(\mathbf{R} / J)-2 d+1,0\} .
$$

\section{UPPER BOUNDS FOR THE VARIATIONS OF $e_{0}(I)$ AND $e_{1}(I)$}

In our calculations we make repeated use of the following elementary observation.

Lemma 2.1. If $(\mathbf{R}, \mathfrak{m})$ is a Noetherian local ring and $M$ is an $\mathbf{R}$-module of finite length $\lambda(M)$, then

$$
\lambda(M \otimes N) \leq \lambda(M) \cdot \nu(N)
$$

for every finitely generated $\mathbf{R}$-module $N$, where $\nu(N)$ denotes the minimal number of generators for $N$.

Proof. Induct on $n=\lambda(M)$. If $n=1$, then $M \simeq \mathbf{R} / \mathfrak{m}$, and the assertion is clear. Suppose that $n \geq 2$ and choose an $\mathbf{R}$-submodule $L$ of $M$ with $\lambda(L)=1$. By tensoring $0 \rightarrow L \rightarrow M \rightarrow M / L \rightarrow 0$ with $N$, we get the exact sequence

$$
L \otimes N \rightarrow M \otimes N \rightarrow(M / L) \otimes N \rightarrow 0 .
$$

Since $\lambda(M / L)=n-1$, the induction hypothesis shows

$$
\lambda((M / L) \otimes N) \leq(n-1) \nu(N),
$$

so that

$$
\lambda(M \otimes N) \leq \lambda(L \otimes N)+\lambda((M / L) \otimes N) \leq(1+(n-1)) \cdot \nu(N)=\lambda(M) \nu(N) .
$$

Theorem 2.2. Let $(\mathbf{R}, \mathfrak{m})$ be a Noetherian local ring of dimension $d$ and let $J \subset$ $I=(J, h)$ be $\mathfrak{m}$-primary ideals of $\mathbf{R}$. Then

$$
e_{0}(J)-e_{0}(I) \leq \lambda(\mathbf{R} /(J: I)) \cdot f_{0}(J) .
$$


Proof. For $n \in \mathbb{N}$, consider the following filtration:

$$
\begin{aligned}
J^{n}=M_{0} \subset M_{1}=\left(M_{0}, J^{n-1} h\right) \subset \cdots & \subset M_{r-1}=\left(M_{r-2}, J^{n-r+1} h^{r-1}\right) \\
& \subset M_{r}=\left(M_{r-1}, J^{n-r} h^{r}\right) \\
& \subset \cdots \subset M_{n}=\left(M_{n-1}, h^{n}\right)=I^{n} .
\end{aligned}
$$

Then we obtain

$$
\lambda\left(\mathbf{R} / J^{n}\right)-\lambda\left(\mathbf{R} / I^{n}\right)=\lambda\left(I^{n} / J^{n}\right)=\lambda\left(M_{n} / M_{0}\right)=\sum_{r=1}^{n} \lambda\left(M_{r} / M_{r-1}\right) .
$$

For each $r, M_{r} / M_{r-1}$ is generated by the image of $h^{r} J^{n-r}+M_{r-1}$. Consider the natural surjection

$$
\zeta: \mathbf{R} /(J: h) \otimes J^{n-r} \rightarrow M_{r} / M_{r-1}=\left(h^{r} J^{n-r}+M_{r-1}\right) / M_{r-1} .
$$

Using Lemma 2.1, we have

$$
\lambda\left(M_{r} / M_{r-1}\right) \leq \lambda\left(\mathbf{R} /(J: h) \otimes J^{n-r}\right) \leq \lambda(\mathbf{R} /(J: h)) \cdot \nu\left(J^{n-r}\right) .
$$

It follows that

$$
\lambda\left(\mathbf{R} / J^{n}\right)-\lambda\left(\mathbf{R} / I^{n}\right) \leq \lambda(\mathbf{R} /(J: I)) \cdot \sum_{r=0}^{n-1} \nu\left(J^{r}\right) .
$$

The iterated Hilbert function $\sum_{r=0}^{n-1} \nu\left(J^{r}\right)$ is of polynomial type of degree $d$ with leading (binomial) coefficient $f_{0}(J)$. Also, for $n \gg 0, \lambda\left(\mathbf{R} / J^{n}\right)-\lambda\left(\mathbf{R} / I^{n}\right)$ is the difference of two polynomials of degree $d$ and leading (binomial) coefficients $e_{0}(J)$ and $e_{0}(I)$. Hence

$$
e_{0}(J)-e_{0}(I) \leq \lambda(\mathbf{R} /(J: I)) \cdot f_{0}(J)
$$

Theorem 2.3. Let $(\mathbf{R}, \mathfrak{m})$ be a Noetherian local ring of dimension $d \geq 1$ and let $J \subset I=(J, h)$ be $\mathfrak{m}$-primary ideals. If $h$ is integral over $J$, then

$$
e_{1}(I)-e_{1}(J) \leq \operatorname{red}_{J}(I) \cdot \lambda(\mathbf{R} /(J: I)) \cdot f_{0}(J),
$$

where $\operatorname{red}_{J}(I)$ is the reduction number of $I$ with respect to $J$.

Proof. Let $s=\operatorname{red}_{J}(I)$. Then $h^{s+1} \in J I^{s}$. For $n \geq s$, we obtain the following filtration:

$$
\begin{aligned}
J^{n}=M_{0} \subset M_{1}=\left(M_{0}, J^{n-1} h\right) \subset \cdots & \subset M_{r}=\left(M_{r-1}, J^{n-r} h^{r}\right) \\
& \subset \cdots \subset M_{s}=\left(M_{s-1}, J^{n-s} h^{s}\right)=I^{n} .
\end{aligned}
$$

Therefore

$$
\lambda\left(\mathbf{R} / J^{n}\right)-\lambda\left(\mathbf{R} / I^{n}\right)=\lambda\left(I^{n} / J^{n}\right)=\sum_{r=1}^{s} \lambda\left(M_{r} / M_{r-1}\right) \leq \lambda(\mathbf{R} /(J: I)) \sum_{r=1}^{s} \nu\left(J^{n-r}\right) .
$$

Now for $n \gg 0, \lambda\left(\mathbf{R} / J^{n}\right)-\lambda\left(\mathbf{R} / I^{n}\right)$ is the difference of two polynomials of degree $d$ and with same leading (binomial) coefficients $e_{0}(J)$ and $e_{0}(I)$. Therefore 
it is at most a polynomial of degree $d-1$ and leading coefficient $e_{1}(I)-e_{1}(J)$. On the other hand, for $n \gg 0$, we have

$$
\lambda(\mathbf{R} /(J: h)) \cdot \sum_{r=1}^{s} \nu\left(J^{n-r}\right) \leq \lambda(\mathbf{R} /(J: h)) \cdot s \cdot \sum_{i=0}^{d-1}(-1)^{i} f_{i}(J)\left(\begin{array}{c}
n+d-i-2 \\
d-i-1
\end{array}\right),
$$

which proves that

$$
e_{1}(I)-e_{1}(J) \leq \operatorname{red}_{J}(I) \cdot \lambda(\mathbf{R} /(J: I)) \cdot f_{0}(J) .
$$

Corollary 2.4. Let $(\mathbf{R}, \mathfrak{m})$ be a Noetherian local ring of dimension $d \geq 1$ and infinite residue field. Let $Q \subset I=(Q, h)$ be $\mathfrak{m}$-primary ideals such that $Q$ is a minimal reduction of $I$. Then

$$
e_{1}(I) \leq \operatorname{red}_{Q}(I) \cdot \lambda(\mathbf{R} /(Q: I)) .
$$

Moreover, if $\mathbf{R}$ is Gorenstein, then

$$
e_{1}(I) \leq \operatorname{red}_{Q}(I) \cdot\left(e_{0}(I)-\lambda(\mathbf{R} / I)\right) .
$$

Proof. The first assertion follows from $e_{1}(Q) \leq 0$ MSV and $f_{0}(Q)=1$ for every parameter ideal $Q$. Suppose that $\mathbf{R}$ is Gorenstein. Then it is enough to show that

$$
\lambda(\mathbf{R} /(Q: I))=e_{0}(I)-\lambda(\mathbf{R} / I) .
$$

This follows from

$\lambda(\mathbf{R} /(Q: I))=\lambda(\mathbf{R} / Q)-\lambda((Q: I) / Q)=e_{0}(Q)-\lambda((Q: I) / Q)=e_{0}(Q)-\lambda(\mathbf{R} / I)$ because $(Q: I) / Q$ is the canonical module of $\mathbf{R} / I$.

Example 2.5 ([V1, Example 7.36]). Let $k[x, y, z]$ be the polynomial ring over an infinite field $k$. Let $\mathbf{R}=k[x, y, z]_{(x, y, z)}$ and let $J$ and $I$ be $\mathbf{R}$-ideals such that

$$
J=\left(x^{a}, y^{b}, z^{c}\right) \subset\left(J, x^{\alpha} y^{\beta} z^{\gamma}\right)=I,
$$

where $\frac{\alpha}{a}+\frac{\beta}{b}+\frac{\gamma}{c}<1$. This inequality ensures that $h=x^{\alpha} y^{\beta} z^{\gamma} \notin \bar{J}$. Then we have

$$
e_{0}(J)-e_{0}(I)=a b c-(a b \gamma+b c \alpha+a c \beta)=a b c\left(1-\frac{\alpha}{a}-\frac{\beta}{b}-\frac{\gamma}{c}\right) .
$$

Since $(J: I)=\left(J: x^{\alpha} y^{\beta} z^{\gamma}\right)=\left(x^{a-\alpha}, y^{b-\beta}, z^{c-\gamma}\right)$, we obtain

$$
\begin{aligned}
\lambda(\mathbf{R} /(J: I)) \cdot f_{0}(J) & =(a-\alpha)(b-\beta)(c-\gamma) \\
& =a b c-b c \alpha-a c \beta-a b \gamma+a \beta \gamma+b \alpha \gamma+c \alpha \beta-\alpha \beta \gamma \\
& =e_{0}(J)-e_{0}(I)+\alpha \beta \gamma\left(\frac{a}{\alpha}+\frac{b}{\beta}+\frac{c}{\gamma}-1\right) \\
& >e_{0}(J)-e_{0}(I) .
\end{aligned}
$$

Let $Q=\left(x^{a}-z^{c}, y^{b}-z^{c}, x^{\alpha} y^{\beta} z^{\gamma}\right)$ and suppose that $a>3 \alpha, b>3 \beta, c>3 \gamma$. Note that $I=\left(Q, z^{c}\right)$. Then $Q$ is a minimal reduction of $I$ and the reduction number $\operatorname{red}_{Q}(I) \leq 2$. We can estimate $e_{1}(I)$ :

$$
e_{1}(I)=e_{1}(I)-e_{1}(Q) \leq 2 \lambda(\mathbf{R} /(Q: I)) \text {. }
$$


Now we treat a general case of Theorem 2.3 Let $J$ be an $\mathfrak{m}$-primary ideal and $H=\left(h_{1}, \ldots, h_{m}\right)$ a set of elements integral over $J$. Write $I=(J, H)$, where $\nu(H)=\nu(I / J)$, and consider the difference of Hilbert functions

$$
\begin{aligned}
\lambda\left(\mathbf{R} / J^{n}\right)-\lambda\left(\mathbf{R} / I^{n}\right) & =\lambda\left((J, H)^{n} / J^{n}\right)=\lambda\left(\left(J^{n}, H J^{n-1}, \ldots, H^{n-1} J, H^{n}\right) / J^{n}\right) \\
& =\sum_{r=1}^{n} \lambda\left(M_{r} / M_{r-1}\right),
\end{aligned}
$$

where $M_{r}=\left(J^{n}, H J^{n-1}, \ldots, H^{r-1} J^{n-r+1}, H^{r} J^{n-r}\right)$. Note that $M_{r} / M_{r-1}$ is generated by the image of $H^{r} J^{n-r}$. More precisely, if $I=\left(J, h_{1}, \ldots, h_{m}\right)$, then $M_{r} / M_{r-1}$ is generated by batches of elements, difficult to control. This filtration has been used by several authors when $J$ is generated by a system of parameters. As $\nu\left(I^{n} / J^{n}\right)$ is increasing, the method of iterating the assertion in Theorem 2.3 tends to induce a larger upper bound for $e_{1}(I)-e_{1}(J)$ than necessary. Instead, our formulation using the filtration above wraps it differently to accommodate our data.

Theorem 2.6. Let $(\mathbf{R}, \mathfrak{m})$ be a Noetherian local ring of dimension $d \geq 1$, let $J$ be an $\mathfrak{m}$-primary ideal and let $I=\left(J, h_{1}, \ldots, h_{m}\right)$ be integral over $J$ of reduction number $s=\operatorname{red}_{J} I$. Then

$$
e_{1}(I)-e_{1}(J) \leq \lambda(\mathbf{R} /(J: I)) \cdot\left[\left(\begin{array}{c}
m+s \\
s
\end{array}\right)-1\right] \cdot f_{0}(J) .
$$

Proof. We have already given parts of the proof. The remaining part is to estimate the growth of the length of $M_{r} / M_{r-1}=\left[\left(h_{1}, \ldots, h_{m}\right)^{r} J^{n-r}+M_{r-1}\right] / M_{r-1}$. We note that this module is annihilated by $J: I$ and is generated by the 'monomials' in the $h_{i}$ of degree $r$, with coefficients in $J^{n-r}$. There is a natural surjection

$$
\Phi: \mathbf{R} /(J: I) \otimes \mathbf{R}^{b_{r}} \otimes J^{n-r} \longrightarrow M_{r} / M_{r-1},
$$

where $b_{r}=\left(\begin{array}{c}m+r-1 \\ r\end{array}\right)$. Therefore for $n \gg 0$,

$$
\begin{aligned}
\lambda\left(\mathbf{R} / J^{n}\right)-\lambda\left(\mathbf{R} / I^{n}\right) & =\sum_{r=1}^{s} \lambda\left(M_{r} / M_{r-1}\right) \\
& \leq \sum_{r=1}^{s} \lambda(\mathbf{R} /(J: I)) \cdot \nu\left(J^{n}\right) \cdot\left(\begin{array}{c}
m+r-1 \\
r
\end{array}\right) \\
& =\lambda(\mathbf{R} /(J: I)) \cdot \nu\left(J^{n}\right) \cdot\left[\left(\begin{array}{c}
m+s \\
s
\end{array}\right)-1\right],
\end{aligned}
$$

which completes the proof.

In Theorem 2.6. if $J$ is a minimal reduction of $I$, then it is well-known that $m=\nu(I)-\nu(J)$ does not depend on $J$ because $\mathfrak{m} I \cap J=\mathfrak{m} J$. Moreover, if $\mathbf{R}$ is Cohen-Macaulay, then $\lambda(\mathbf{R} /(J: I))$ does not depend on $J$ either, because $\lambda(\mathbf{R} /(J: I))=e_{0}(I)-\lambda\left(\mathrm{H}_{m}(I)\right)$, where $\mathrm{H}_{m}(I)$ is the $m$-th Koszul homology of $I$.

Proposition 2.7. Let $(\mathbf{R}, \mathfrak{m})$ be a Noetherian local ring of dimension $d \geq 1$, let $J$ be an $\mathfrak{m}$-primary ideal and let $I=\left(J, h_{1}, \ldots, h_{m}\right)$ be integral over $J$ of reduction 
number $s=\operatorname{red}_{J} I$. Then

$$
f_{0}(I) \leq\left(1+\lambda(\mathbf{R} /(J: I)) \cdot\left[\left(\begin{array}{c}
m+s \\
s
\end{array}\right)-1\right]\right) \cdot f_{0}(J) .
$$

Proof. By tensoring the following exact sequence with $\mathbf{R} / \mathfrak{m}$,

$$
0 \longrightarrow J^{n} \longrightarrow I^{n} \longrightarrow I^{n} / J^{n} \longrightarrow 0,
$$

we obtain

$$
J^{n} / \mathfrak{m} J^{n} \longrightarrow I^{n} / \mathfrak{m} I^{n} \longrightarrow\left(I^{n} / J^{n}\right) \otimes \mathbf{R} / \mathfrak{m} \rightarrow 0 .
$$

Therefore, using Lemma 2.1, we get

$$
\begin{aligned}
\lambda\left(I^{n} / \mathfrak{m} I^{n}\right)-\lambda\left(J^{n} / \mathfrak{m} J^{n}\right) & \leq \lambda\left(\left(I^{n} / J^{n}\right) \otimes \mathbf{R} / \mathfrak{m}\right) \\
& \leq \lambda\left(I^{n} / J^{n}\right)=\lambda\left(\mathbf{R} / J^{n}\right)-\lambda\left(\mathbf{R} / I^{n}\right) .
\end{aligned}
$$

This induces the inequalities of the leading coefficients (in degree $d-1$ )

$$
f_{0}(I)-f_{0}(J) \leq e_{1}(I)-e_{1}(J) .
$$

Using Theorem 2.6, we obtain

$$
f_{0}(I)-f_{0}(J) \leq e_{1}(I)-e_{1}(J) \leq \lambda(\mathbf{R} /(J: I)) \cdot\left[\left(\begin{array}{c}
m+s \\
s
\end{array}\right)-1\right] \cdot f_{0}(J),
$$

which completes the proof.

Remark 2.8. Note that the formulas for the variations of $e_{1}(I)$ and $f_{0}(I)$ require that the ideal $I$ have the same integral closure as $J$.

The values of the first Hilbert coefficients are also related to the multiplicity of certain Sally modules, according to [C, Proposition 2.8]. Let $(\mathbf{R}, \mathfrak{m})$ be a Noetherian local ring of dimension $d \geq 1$ with infinite residue field. Let $I$ be an $\mathfrak{m}$-primary ideal and $Q$ a minimal reduction of $I$. If $\operatorname{dim}\left(S_{Q}(I)\right)=d$ and $H_{\mathfrak{m}}^{0}(\mathbf{R}) \subset I$, then the multiplicity $s_{0}(Q, I)$ of the Sally module $S_{Q}(I)$ is

$$
s_{0}(Q, I)=e_{1}(I)-e_{1}(Q)-e_{0}(I)+\lambda(\mathbf{R} / I) .
$$

Corollary 2.9. Let $(\mathbf{R}, \mathfrak{m})$ be a Noetherian local ring of dimension $d \geq 1$ with infinite residue field. Let $I$ be an $\mathfrak{m}$-primary ideal and $Q$ a minimal reduction of $I$. Suppose that $\operatorname{dim}\left(S_{Q}(I)\right)=d$ and that $H_{\mathfrak{m}}^{0}(\mathbf{R}) \subset I$. Then the multiplicity $s_{0}(Q, I)$ of the Sally module $S_{Q}(I)$ satisfies

$$
s_{0}(Q, I) \leq-e_{0}(I)+\lambda(\mathbf{R} / I)+\lambda(\mathbf{R} /(Q: I)) \cdot\left[\left(\begin{array}{c}
\nu(I)-d+s \\
s
\end{array}\right)-1\right],
$$

where $s=\operatorname{red}_{Q}(I)$ is the reduction number.

Example 2.10. Let $\mathbf{R}=k[x, y]_{(x, y)}$, where $k[x, y]$ denotes the polynomial ring over an infinite field $k$. Let $\mathfrak{m}=(x, y)$ and $I=\mathfrak{m}^{n}=\left(a_{1}, \ldots, a_{n}, a_{n+1}\right)$ for some $n \geq 2$. We assume that $Q=\left(a_{1}, a_{2}\right)$ is a minimal reduction of $I$. Let $J=\left(a_{1}, a_{2}, \ldots, a_{n}\right)$. Then since $Q \subseteq J, I$ is integral over $J$ with $\operatorname{red}_{J}(I)=1$, because $I \neq J$ and $\operatorname{red}_{Q}(I)=1$. Using $e_{1}(I)=e_{1}\left(\mathfrak{m}^{n}\right)=\frac{1}{2} n(n-1)$ and

$$
e_{1}(I)-e_{1}(J) \leq \lambda\left(\mathbf{R} /\left(J: a_{n+1}\right)\right) f_{0}(J),
$$

we obtain

$$
e_{1}(J) \geq \frac{1}{2} n(n-1)-\lambda\left(\mathbf{R} /\left(J: a_{n+1}\right)\right) f_{0}(J)
$$


One situation that may be amenable to further analysis is when $I=J: \mathfrak{m}$ or, more generally, when $I=J: \mathfrak{m}^{s}$ for some values of $s$. We refer to $I$ as a socle extension of $J$.

Remark 2.11 (Reduction number one). Let $(\mathbf{R}, \mathfrak{m})$ be a Noetherian local ring of dimension $d \geq 1$ with infinite residue field. Let $I$ be an $\mathfrak{m}$-primary ideal and $Q$ a minimal reduction of $I$. Suppose that $I^{2}=Q I$. Then by Theorem 2.6 we get

$$
e_{1}(I)-e_{1}(Q) \leq \lambda(\mathbf{R} /(Q: I)) \cdot(\nu(I)-d) \leq \lambda(\mathbf{R} /(Q: I)) \cdot \lambda(I / Q) .
$$

Suppose that $\mathbf{R}$ is Cohen-Macaulay. Then since $e_{1}(I)=e_{0}(I)-\lambda(\mathbf{R} / I)=\lambda(I / Q)$ $([\mathrm{Hu}, 2.1])$, it follows that

$$
e_{1}(I)=e_{1}(I)-e_{1}(Q) \leq \lambda(\mathbf{R} /(Q: I)) \cdot \lambda(I / Q)=\lambda(\mathbf{R} /(Q: I)) \cdot e_{1}(I) .
$$

For example, if $\mathbf{R}$ is a Cohen-Macaulay local ring that is not regular and $I=Q: \mathfrak{m}$, then

$$
e_{1}(I)=e_{1}(I)-e_{1}(Q) \leq \lambda(\mathbf{R} /(Q: I)) \cdot(\nu(I)-d) \leq \lambda(I / Q)=e_{1}(I),
$$

which is a case when the equality in Theorem 2.6 holds true.

Example 2.12. Let $\mathbf{R}$ be a Cohen-Macaulay local ring of dimension 1 with $e_{0}(\mathbf{R})=2$. For every $\mathfrak{m}$-primary ideal $I$, there exists $a \in I$ such that $I^{2}=a I$ ([SV, Theorem 2.5]). Hence

$$
e_{1}(I)=\lambda(\mathbf{R} /(a \mathbf{R}: I)) \cdot[\nu(I)-1] .
$$

Example 2.13. Let $a$ and $\ell$ be integers such that $a \geq 4$ and $\ell \geq 2$. Let $H$ be the numerical semigroup generated by $a$, al $-1,\{a \ell+i\}_{1 \leq i \leq a-3}$, and put $\mathbf{R}=$ $k\left[\left[t^{a}, t^{a \ell-1},\left\{t^{a \ell+i}\right\}_{1 \leq i \leq a-3}\right]\right]$ in the formal power series ring $k[[t]]$ over a field $k$. Let $I=\left(t^{2 a \ell-a-1},\left\{t^{3 a \ell-2 a-1-i}\right\}_{1 \leq i \leq a-3}\right) \subsetneq \mathbf{R}$ and $Q=\left(t^{2 a \ell-a-1}\right) \subset I$. Then $I=\omega_{\mathbf{R}}$ is a canonical ideal of $\mathbf{R}$ and $Q$ is a reduction of $I$. We have

$$
\mathfrak{m} I \subseteq Q \text { and } e_{1}(I)=\lambda\left(\mathbf{R}\left[t^{a \ell-a-i} \mid 1 \leq i \leq a-3\right] / \mathbf{R}\right)=a-2=r(\mathbf{R})
$$

(GMP, Lemma 2.1]), where $r(\mathbf{R})$ is the Cohen-Macaulay type. Hence

$$
a-3=r(\mathbf{R})-1=\nu(I / Q)=\lambda(I / Q)<e_{1}(I)=a-2 .
$$

Since $r(\mathbf{R}) \geq 2$, the ring $\mathbf{R}$ is not Gorenstein. We have $e_{1}(I)=\lambda(I / Q)+1$ so that

$$
e_{1}(I)=e_{0}(I)-\lambda(\mathbf{R} / I)+1 .
$$

Therefore, thanks to $\left[\mathrm{S} 2\right.$, we get $I^{3}=Q I^{2}$ (hence $\left.\operatorname{red}_{Q}(I)=2\right)$ and

$$
S_{Q}(I) \simeq B(-1)
$$

as graded $\mathcal{R}(Q)$-modules, where $\mathcal{R}(Q)$ denotes the Rees algebra of $Q, S_{Q}(I)$ the Sally module of $I$ with respect to $Q$, and $B=\mathcal{R}(Q) / \mathfrak{m} \mathcal{R}(Q)$. We have

$$
a-2=e_{1}(I) \leq \lambda(\mathbf{R} /(Q: I))\left[\left(\begin{array}{c}
m+s \\
s
\end{array}\right)-1\right]=\left(\begin{array}{c}
a-1 \\
2
\end{array}\right)-1,
$$

since $s=2$ and $m=a-3$. The equality $e_{1}(I)=\lambda(\mathbf{R} /(Q: I))\left[\left(\begin{array}{c}m+s \\ s\end{array}\right)-1\right]$ holds if and only if $a=4$. When this is the case, we have $H=\langle 4,4 \ell-1,4 \ell+1\rangle$. 


\section{The REDUCTION Number FORMUla}

In order to make use of Theorem 2.6, we need information about the reduction number of $I$ in terms related to multiplicity. Let us recall [V1, Theorem 2.45]:

Theorem 3.1. Let $(\mathbf{R}, \mathfrak{m})$ be a Cohen-Macaulay local ring of dimension $d \geq 1$ and infinite residue field. For an $\mathfrak{m}$-primary ideal $I$,

$$
\operatorname{red}(I) \leq \max \left\{\frac{d \cdot e_{0}(I)}{o(I)}-2 d+1,0\right\}
$$

where $o(I)$ is the $\mathfrak{m}$-adic order of $I$.

To establish such a result for arbitrary Noetherian local rings, we proceed differently. The version of the following lemma for Cohen-Macaulay rings can be found in [S1, Chapter 3, Theorem 1.1].

Lemma 3.2. Let $(\mathbf{R}, \mathfrak{m})$ be a Noetherian local ring of dimension 1 . Let $x$ be a parameter of $\mathbf{R}$. Let $E$ be a finitely generated $\mathbf{R}$-module and $U$ an $\mathbf{R}$-submodule of $E$. Then we have the following:

(a) $\nu(U) \leq \lambda(\mathbf{R} /(x)) \cdot \nu(E)$. Hence $\nu(I) \leq \lambda(\mathbf{R} /(x))$ for every ideal $I$ of $\mathbf{R}$.

(b) If $\mathbf{R}$ is Cohen-Macaulay and $x$ belongs to $\mathfrak{m}^{s}$, then $\nu(U) \leq \frac{\lambda(\mathbf{R} /(x))}{s} \cdot \nu(E)$.

Proof. (a) Let $W=\mathrm{H}_{\mathfrak{m}}^{0}(E), E^{\prime}=E / W$, and $U^{\prime}=(U+W) / W$. Then $E^{\prime}$ is a Cohen-Macaulay $\mathbf{R}$-module of dimension 1 and $x$ is $E^{\prime}$-regular. Moreover,

$$
\lambda\left(U^{\prime} / x U^{\prime}\right)=e_{0}\left((x), U^{\prime}\right) \leq e_{0}\left((x), E^{\prime}\right)=\lambda\left(E^{\prime} / x E^{\prime}\right) .
$$

Consider the following two short exact sequences:

Then we obtain

$$
\begin{aligned}
& 0 \longrightarrow W \longrightarrow E \longrightarrow W \longrightarrow E^{\prime} \longrightarrow 0, \\
& 0 \longrightarrow U \longrightarrow U^{\prime} \longrightarrow 0 .
\end{aligned}
$$

$$
\begin{aligned}
\nu(U) \leq \lambda(U / x U) & =\lambda\left(U^{\prime} / x U^{\prime}\right)+\lambda((U \cap W) / x(U \cap W)) \\
& =\lambda\left(U^{\prime} / x U^{\prime}\right)+\lambda\left(\left(0:_{U \cap W} x\right)\right) \\
& \leq \lambda\left(U^{\prime} / x U^{\prime}\right)+\lambda\left(\left(0:_{W} x\right)\right) \\
& =\lambda\left(U^{\prime} / x U^{\prime}\right)+\lambda(W / x W) \\
& \leq \lambda\left(E^{\prime} / x E^{\prime}\right)+\lambda(W / x W) \\
& =\lambda(E / x E) \\
& \leq \lambda(\mathbf{R} /(x)) \cdot \nu(E) .
\end{aligned}
$$

(b) We may assume that the field $\mathbf{R} / \mathfrak{m}$ is infinite. Let $y \mathbf{R}$ be a minimal reduction of $\mathfrak{m}$. Then since $x \in \mathfrak{m}^{s} \subseteq \overline{y^{s} \mathbf{R}}$, we get

$$
\lambda(\mathbf{R} /(x))=e_{0}(x \mathbf{R}) \geq e_{0}\left(\overline{y^{s} \mathbf{R}}\right)=e_{0}\left(y^{s} \mathbf{R}\right)=s \cdot e_{0}(y \mathbf{R})=s \cdot \lambda(\mathbf{R} /(y)) .
$$

Hence $\lambda(\mathbf{R} /(y)) \leq \frac{\lambda(\mathbf{R} /(x))}{s}$ so that

$$
\nu(U) \leq \lambda(\mathbf{R} /(y)) \cdot \nu(E) \leq \frac{\lambda(\mathbf{R} /(x))}{s} \cdot \nu(E) .
$$


Theorem 3.3. Let $(\mathbf{R}, \mathfrak{m})$ be a Noetherian local ring of dimension $d \geq 1$ with infinite residue field. For an $\mathfrak{m}$-primary ideal $I$ and a minimal reduction $J$ of $I$, there exists a minimal reduction $Q$ of I such that

$$
\operatorname{red}_{Q}(I) \leq \max \{d \cdot \lambda(\mathbf{R} / J)-2 d+1,0\} .
$$

Proof. Let us start with a minimal reduction $J=\left(x_{1}, \ldots, x_{d}\right)$ of $I$. Let $L=$ $\left(x_{1}, \ldots, x_{d-1}\right)$. Then

$$
\nu\left(I^{n}\right) \leq \nu\left(L^{n}\right)+\nu\left(I^{n} / L^{n}\right) .
$$

We need to estimate $\nu\left(I^{n} / L^{n}\right)$. Set

$$
M_{i}=\frac{\left(I^{n}+L^{n-i+1}\right) \cap L^{n-i}}{L^{n-i+1}} \text { and } \quad N_{i}=\frac{I^{n}+L^{n-i+1}}{L^{n-i+1}} .
$$

Then we obtain the following series of exact sequences:

$$
0 \longrightarrow M_{i} \longrightarrow N_{i} \longrightarrow N_{i+1} \longrightarrow 0,
$$

where $i=1, \ldots, n-1$. Note that for each $i, M_{i}$ is a submodule of $L^{n-i} / L^{n-i+1}$ as an $\mathbf{R} / L$-module. Hence by Lemma 3.2, for each $i=1, \ldots, n-1$,

$$
\nu\left(M_{i}\right) \leq \lambda(\mathbf{R} / J) \cdot \nu\left(L^{n-i} / L^{n-i+1}\right)=\lambda(\mathbf{R} / J) \cdot\left(\begin{array}{c}
d+n-2-i \\
d-2
\end{array}\right) .
$$

Since $N_{n}=\frac{I^{n}+L}{L}$ is a submodule of $\mathbf{R} / L$, by Lemma 3.2, we get

$$
\nu\left(N_{n}\right) \leq \lambda(\mathbf{R} / J) .
$$

Therefore we obtain

$$
\begin{aligned}
\nu\left(I^{n}\right) & \leq \nu\left(L^{n}\right)+\nu\left(I^{n} / L^{n}\right) \\
& \leq \nu\left(L^{n}\right)+\nu\left(M_{1}\right)+\nu\left(M_{2}\right)+\cdots+\nu\left(M_{n-1}\right)+\nu\left(N_{n}\right) \\
& \leq\left(\begin{array}{c}
d+n-2 \\
d-2
\end{array}\right)+\lambda(\mathbf{R} / J) \cdot \sum_{i=1}^{n}\left(\begin{array}{c}
d+n-2-i \\
d-2
\end{array}\right) \\
& =\left(\begin{array}{c}
d+n-2 \\
d-2
\end{array}\right)+\lambda(\mathbf{R} / J) \cdot\left(\begin{array}{c}
d+n-2 \\
d-1
\end{array}\right) .
\end{aligned}
$$

Recall that if

$$
\nu\left(I^{n}\right)<\left(\begin{array}{c}
n+d \\
d
\end{array}\right)
$$

then there is a minimal reduction $Q$ of $I$ such that $\operatorname{red}_{Q}(I) \leq n-1($ ES], V1, Theorem 2.36]). Hence by solving the inequality

$$
\left(\begin{array}{c}
d+n-2 \\
d-2
\end{array}\right)+\lambda(\mathbf{R} / J)\left(\begin{array}{c}
d+n-2 \\
d-1
\end{array}\right)<\left(\begin{array}{c}
n+d \\
d
\end{array}\right)
$$

we obtain the desired relation.

Corollary 3.4. Let $(\mathbf{R}, \mathfrak{m})$ be a Noetherian local ring of dimension $d \geq 1$ and infinite residue field. Let $Q$ be a minimal reduction of $\mathfrak{m}$ such that $\operatorname{red}_{Q}(\mathfrak{m})=$ $\operatorname{red}(\mathfrak{m})$. Then

$$
e_{1}(\mathfrak{m}) \leq e_{1}(\mathfrak{m})-e_{1}(Q) \leq \lambda(\mathbf{R} /(Q: \mathfrak{m})) \cdot\left[\left(\begin{array}{c}
\nu(\mathfrak{m})+\lambda(\mathbf{R} / Q) d-3 d+1 \\
\nu(\mathfrak{m})-d
\end{array}\right)-1\right] .
$$


Remark 3.5. It is worthwhile to point out that there are other known bounds for the reduction number of an ideal in terms of some of its Hilbert coefficients. One of these is a bound proved by M. E. Rossi ( $[\mathbb{R}$, Corollary 1.5]): If $(\mathbf{R}, \mathfrak{m})$ is a CohenMacaulay local ring of dimension at most 2 , then for any $\mathfrak{m}$-primary ideal $I$ with a minimal reduction $Q$,

$$
\operatorname{red}_{Q}(I) \leq e_{1}(I)-e_{0}(I)+\lambda(\mathbf{R} / I)+1 .
$$

Several open questions arise. Foremost is whether it extends to higher dimensional Cohen-Macaulay rings (with a correction term depending on the dimension). Another question is which offsetting terms should be added in the non-CohenMacaulay case; for instance, it would be interesting to know whether the addition of $-e_{1}(Q)$, a term that can be considered a non-Cohen-Macaulayness penalty, would give a valid bound in a 2-dimensional ring.

\section{Normalization}

The following observation shows how the special fiber of the normalization impacts $e_{0}(I)$. Of course, a more interesting issue would be to obtain relationships going the other way.

Proposition 4.1. Let $(\mathbf{R}, \mathfrak{m})$ be a normal local domain and let $I$ be an $\mathfrak{m}$-primary ideal. Suppose that $\overline{\mathcal{R}}=\bigoplus_{n=0}^{\infty} C_{n}$ is finite over $\mathcal{R}=\mathcal{R}(I)$. We denote by $\bar{f}_{0}(I)$ the multiplicity of $\overline{\mathcal{R}} / \mathfrak{m} \overline{\mathcal{R}}$. Then

$$
e_{0}(I) \leq \min \left\{f_{0}(I) \cdot \lambda(\mathbf{R} / I), \quad \bar{f}_{0}(I) \cdot \lambda(\mathbf{R} / \bar{I})\right\} .
$$

Proof. We first observe that $C_{n+1}=I C_{n}=\bar{I} C_{n}$, for $n \gg 0$. In particular, in that range, $C_{n+1} \subset \mathfrak{m} C_{n}$. Now consider the corresponding exact sequence:

$$
0 \rightarrow \mathfrak{m} C_{n} / C_{n+1} \longrightarrow C_{n} / C_{n+1} \longrightarrow C_{n} / \mathfrak{m} C_{n} \rightarrow 0 .
$$

Counting multiplicities, we have

$$
e_{0}(I) \leq \operatorname{deg}(\mathfrak{m} \overline{\mathcal{R}} / \bar{I} \overline{\mathcal{R}})+\operatorname{deg}(\overline{\mathcal{R}} / \mathfrak{m} \overline{\mathcal{R}}) \leq \bar{f}_{0}(I)(\lambda(\mathfrak{m} / \bar{I})+1)=\bar{f}_{0}(I) \cdot \lambda(\mathbf{R} / \bar{I}),
$$

as desired. The other inequality, $e_{0}(I) \leq f_{0}(I) \cdot \lambda(\mathbf{R} / I)$, has a similar proof.

\section{REFERENCES}

[C] A. Corso, Sally modules of m-primary ideals in local rings, Comm. Algebra 37 (2009), 4503-4515. MR2588863(2011e:13007)

[ES] P. Eakin and A. Sathaye, Prestable ideals, J. Algebra 41 (1976), 439-454. MR0419428 (54:7449)

[E1] J. Elias, On the first normalized Hilbert coefficient, J. Pure and Applied Algebra 201 (2005), 116-125. MR2158750 (2006d:13017)

[E2] J. Elias, Upper bounds of Hilbert coefficients and Hilbert functions, Math. Proc. Camb. Phil. Soc. 145 (2008), 87-94. MR2431640 (2009d:13020)

[GhGHOPV] L. Ghezzi, S. Goto, J. Hong, K. Ozeki, T.T. Phuong and W. V. Vasconcelos, CohenMacaulayness versus the vanishing of the first Hilbert coefficient of parameter ideals, J. London Math. Soc. 81 (2010), 679-695. MR2650791 (2011k:13040)

[GhHV] L. Ghezzi, J. Hong and W. V. Vasconcelos, The signature of the Chern coefficients of local rings, Math. Research Letters 16 (2009), 279-289. MR2496744 (2010b:13003)

[GHM] S. Goto, J. Hong and M. Mandal, The positivity of the first normalized Hilbert coefficients, Proc. Amer. Math. Soc. 139 (2011), 2399-2406. MR2784804(2012e:13031)

[GMP] S. Goto, N. Matsuoka and T. T. Phuong, Almost Gorenstein rings, preprint. arXiv:1106.1301v2 [Math.AC]. 
[GO] S. Goto and K. Ozeki, Buchsbaumness in local rings possessing constant first Hilbert coefficients of parameters, Nagoya Math. J. 199 (2010), 95-105. MR 2730412 (2011m:13048)

[HH] K. Hanumanthu and C. Huneke, Bounding the first Hilbert coefficient, Proc. Amer. Math. Soc. 140 (2012), 109-117. MR2833522(2012e:13032)

[Hu] C. Huneke, Hilbert functions and symbolic powers, Michigan Math. J. 34 (1987), 293-318. MR894879 (89b:13037)

[K] D. Kirby, A note on superficial elements of an ideal of a local ring, Q. J. Math. Oxford 14 (1963), 21-28. MR0143780(26:1332)

[MV] M. Mandal and J. K. Verma, On the Chern number of an ideal, Proc. Amer. Math. Soc. 138 (2010), 1995-1999. MR2596035 (2011c:13029)

[MSV] M. Mandal, B. Singh and J. K. Verma, On some conjectures about the Chern numbers of filtrations, J. Algebra 325 (2011), 147-162. MR2745533 (2012a:13028)

[PUV] C. Polini, B. Ulrich and W. V. Vasconcelos, Normalization of ideals and BriançonSkoda numbers, Math. Research Letters 12 (2005), 827-842. MR2189243 (2006m:13006)

[R] M. E. Rossi, A bound on the reduction number of a primary ideal, Proc. Amer. Math. Soc. 128 (2000), 1325-1332. MR1670423 (2000j:13004)

[RV1] M. E. Rossi and G. Valla, The Hilbert function of the Rattliff-Rush filtration, J. Pure and Applied Algebra 201 (2005), 24-41. MR2158745 (2006g:13008)

[RV2] M. E. Rossi and G. Valla, On the Chern number of a filtration, Rendiconti Seminario Matematico Padova 121 (2009), 201-222. MR2542142(2010h:13041)

[S1] J. D. Sally, Numbers of Generators of Ideals in Local Rings, Lecture Notes in Pure and Applied Mathematics 36, Marcel Dekker, New York, 1978. MR 0485852 (58:5654)

[S2] J. D. Sally, Hilbert coefficients and reduction number 2, J. Algebraic Geometry 1 (1992), 325-333. MR:1144442 (93b:13026)

[SV] J. D. Sally and W. V. Vasconcelos, Stable rings, J. Pure and Applied Algebra 4 (1974), 319-336. MR0409430 (53:13185)

[V1] W. V. Vasconcelos, Integral Closure, Springer Monographs in Mathematics, Springer, Heidelberg, 2005. MR2153889 (2006m:13007)

[V2] W. V. Vasconcelos, The Chern coefficients of local rings, Michigan Math. J. 57 (2008), 725-743. MR2492478 (2009m:13005)

Department of Mathematics, New York City College of Technology-CUNy, 300 Jay Street, Brooklyn, New York 11201

E-mail address: lghezzi@citytech.cuny.edu

Department of Mathematics, School of Science and Technology, Meiji University, 1-1-1 Higashi-mita, TAMa-KU, KaWASAKi 214-8571, JAPAN

E-mail address: goto@math.meiji.ac.jp

Department of Mathematics, Southern Connecticut State University, 501 Crescent Street, New Haven, Connecticut 06515-1533

E-mail address: hongj2@southernct.edu

Department of Mathematics, Rutgers University, 110 Frelinghuysen Road, PiscatAWAY, NEW JERSEY 08854-8019

E-mail address: vasconce@math.rutgers.edu 